\title{
Psychosocial Care: Emerging Need for the Cancer Patients in Nepal
}

\author{
Shah $\mathrm{T},{ }^{1}$ Subba $\mathrm{S},{ }^{2}$ Shah $\mathrm{SP}^{3}$ \\ ${ }^{1}$ Department of Community Health Nursing, College of Nursing, B.P. Koirala Institute of health sciences, Dharan, \\ 2Department of Psychology, Tribhuvan University, Kirtipur, Kathmandu, ${ }^{3}$ District Health Officer, Sunsari District Hospital, \\ Inaruwa
}

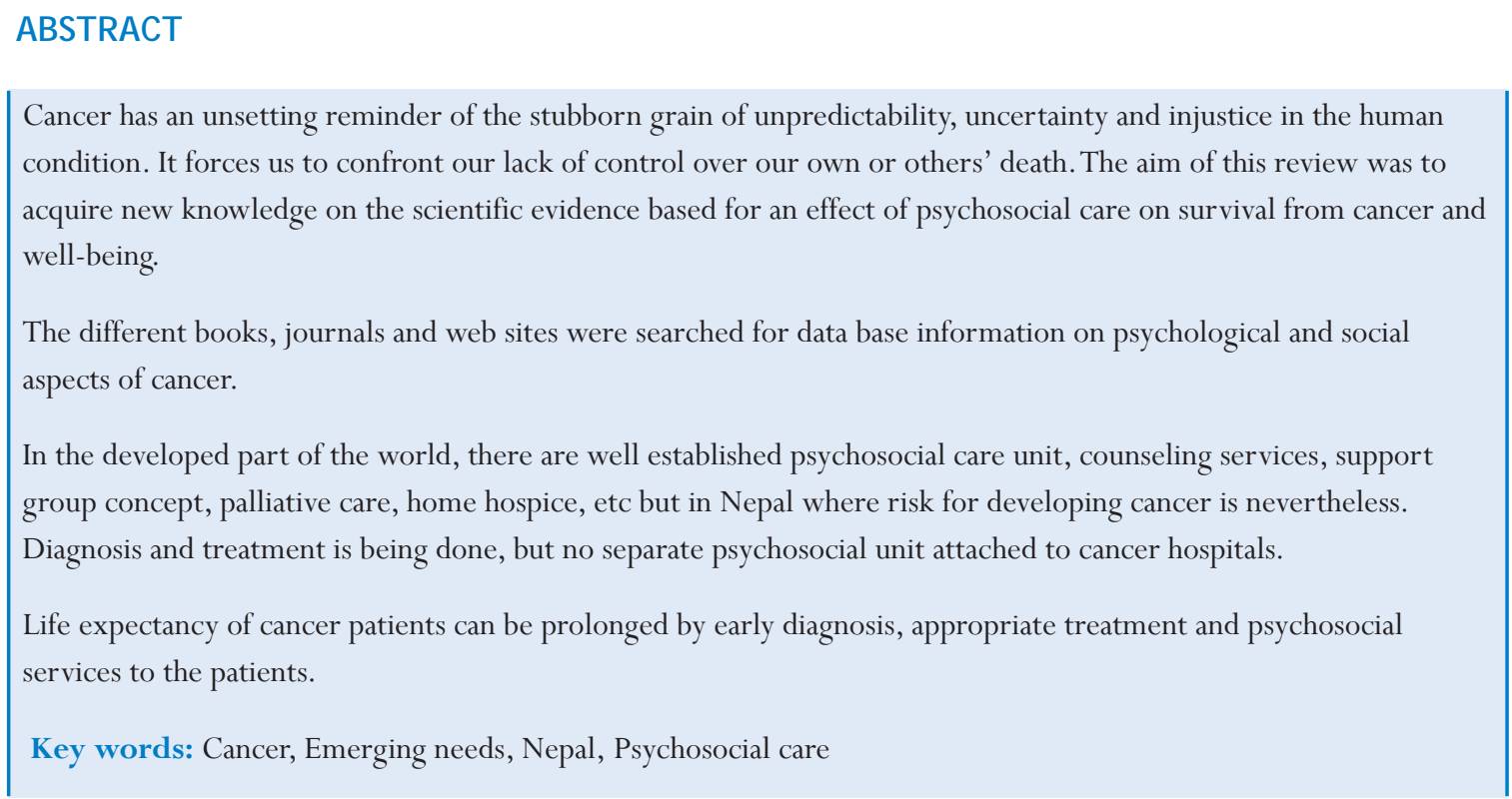

\section{INTRODUCTION}

WHO report showed that cancer is and will become an increasingly important factor in the global burden of the disease in the decades to come. The estimated number of new cases each year is expected to increase from 10 million in 2002 to 15 million by $2020 .{ }^{1}$ Cancer is the second most frequent cause of death in developed countries accounting for $21 \%$ and $7 \%$ of all deaths in the developing countries. ${ }^{2}$ Nepal has no systemic population based cancer registry, the prevalence of the disease is only based on rough estimates which indicate about 50 thousand people suffering from cancer in Nepal. ${ }^{3}$

The connection between the mind and the body is nothing new to those involved in the practice and process of psychology. Stress, a term that represents a variety of emotional states, has been proven to adversely affect the immune system. Chronic repressed emotions and chronic stress can reduce immune function, making a person more susceptible to diseases and distress. Individuals and families who face a diagnosis of cancer experience varying levels of stress and emotional upset. Depression in patients with cancer not only affects the patients themselves but also has a major negative impact on their families.

Cancer has been a dreadful disease of mankind from the beginning of the civilization. When cancer grabs a person, it leaves a life long impact and as some cancers have known genetic link, their offspring also become scared for acquiring the same disease. Stress level is very high amongst those who are associated with cancer, either suffering from the disease or a family member, relative 
Psychosocial Care: Emerging Need for the Cancer Patients in Nepal

or friend of the sufferer. Uncertainty is a common experience that characterizes the life of a cancer patient during the survival period following diagnosis and treatment. Their material conditions of life and their position in the social structure greatly affect their behavior, which in turn affect their health. In addition, influences on behavior, loosely categorized as culture, appear to affect patterns of eating, drinking and social relationship; and may be relatively independent of current material conditions.

IIIness takes meaning as suffering, because of the way this relationship between body and self is mediated by cultural symbols of a religious, moral, or spiritual kind. Suffering encompasses many dimensions which include physical, psychological, social, emotional and spiritual aspects. Being submissive to the belief that it is Karma (destiny, based on one's actions), or God's will, or even a curse, is a frequent way in which South Asian communities deal with the social impact of the disease. Language can be very powerful-social stigmata associated with cancer are so deeply engrained that critical terms are commonly used without taking into consideration the psychological impact it can cause on a cancer survivor. Remarks such as "This problem is spreading like cancer" can be rather disturbing for those who are living with cancer. Communication is another major problem, what to speak or not to speak about it, or whether to make a big deal of it or to ignore it, really makes a difference. People have different fears: about the future and the unknown.

Cancer brings particular cultural meaning to the person. Local cultural orientations organize our conventional common sense about how to understand and treat illness experience that it is always culturally shaped. But conventional expectations about illness are altered through negotiations in different social situations and in particular webs of relationships. ${ }^{4}$ The importance of assessing and understanding the meaning of cancer to the patient and in integrating an awareness of information provision, family support, coping strategy enhancement and using writing to facilitate adjustment are considered. The main evidence-based psychological therapies and their impact on survival are highlighted in psychosocial oncology. ${ }^{5}$ Therefore, there is need to implement the concept of psychosocial care in the health care delivery system of Nepal.

We searched related literatures from 1998-2008; different books, journals, news papers and web sites (Google, HINARI, Blackwell, Science direct, Medline, etc.) were searched with the keywords psychological, social aspects and psychosocial care related to cancer. Citations were compiled manually and reviews were identified. The 16 studies were included, which were related to factors and care associated with psychosocial oncology.

The limited existing literature points to high levels of psychological morbidity, rates of psychological distress from 30 to $45.4 \%$ being reported. A study done in Queensland, Australia among 269 women followed prospectively for three years following the diagnosis of early breast cancer revealed $50 \%$ clinically anxious, depressed, or both. ${ }^{6}$ A survey in England of women with breast cancer showed that among several factors, depression was the strongest predictor of emotional and behavioral problems in their children. ${ }^{7}$ Fear of death, disruption of life plans, changes in body image and self-esteem, changes in social role and lifestyle, and financial and legal concerns are significant issues in the life of any person with cancer, yet serious depression or anxiety is not experienced by everyone who is diagnosed with cancer. J ust as patients require ongoing evaluation for depression and anxiety throughout their course of treatment, so do family caregivers. In a study of family caregivers of patients in the palliative phase of illness, both male and female caregivers experienced significantly more anxiety than normal samples, while there was an increased incidence of hospital anxiety and depression. ${ }^{8}$

Chronic illness brings about debilitating and lifeendangering impacts on the patient. It interferes with his daily activities, earning capacity, his social and familial relationships and his sexual activities. Acute depressions are very frequent, sometimes accompanied by marked suicidal tendencies.

Percentage Distribution related to Suicidality among

Cancer Patients

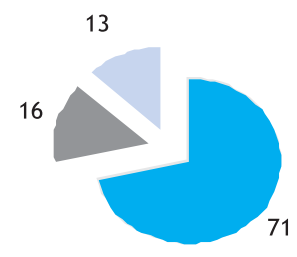

Suicidal ideation Suicidal attempt Requested euthanasia

Figure 1. Percentage distribution related to suicidal tendency among cancer patients

Many epidemiological studies have indicated that the risk of suicide in cancer patients is higher than that of the general population. Figure 1 depicts that of 1713 psychiatric referrals, 62 (3.6\%) were related to suicidality, including 44 cases with suicidal ideation, 10 suicide 
Psychosocial Care: Emerging Need for the Cancer Patients in Nepal

attempts and eight cases who had requested euthanasia and/ or continuous sedation. Most of the patients suffered from physical distress and/ or psychiatric disorders. These findings suggest that suicidal cancer patients suffer from complicated physical and psychological distress. ${ }^{9} \mathrm{~A}$ 50 years male, suffering from colon cancer of Morang district of Nepal committed suicide due to physical distress (pain) and lack of money for treatment. ${ }^{10}$

Cancer raises multiple psychological difficulties not only to the patient and his/her family, but also to the physicians and other members of the care team. Interactions among and between all these people are complex. Psychological support for patients aims at offering harmonious treatment progression and quality of life similar to that experienced before cancer.

DeVries et al revealed that there is a positive correlation between social support and level of health. Social support is implicated to have a buffering effect on stress response, which is closely tied to the endocrine system through the hypothalamic-pituitary-adrenal axis. ${ }^{11}$ Social supports may play a physiologic role in modulation of health through the stress response system. Chronic exposure to psychosocial stress may alter the hypothalamic-pituitary-adrenal axis function. Social support promotes adaptive behavior and modulates the hypothalamic-pituitary-adrenal axis response in the face of stressful situations and the threat of ill health. However, it is difficult to determine the significance of social support on health due largely in part to the multidimensional nature of social support. ${ }^{12}$

Social support in the face of a diagnosis, regarded as a life crisis, can impact the course of the disease, positively or negatively. Cancer patient's need for support is often extended beyond the initial diagnosis and well into the treatment phase and frequently beyond remission. Cancer and its associated treatments may require a fundamental change in lifestyle, which prompts the person to question their personal identity and self worth. It is important to consider the amount and type of social support that will be available as adjustment to this diagnosis made. Literatures addressed that there is very little information addressing negative aspects associated with social support; however it bears noting that these aspects do exist and can greatly impact a person's ability to give and receive support.

The psychosocial issues in persons facing life threatening illness like cancer are influenced by individual, sociocultural, medical and family factors. The diagnosis of cancer indicates the end of life and thoughts turn to death and dying with the sudden realization that death is waiting around the corner. Living with a diagnosis of cancer can create a great deal of emotional stress. Treatment, the fear of a relapse, and generalized distress that results from living with the day-to-day physical problems often associated with a cancer diagnosis can create new or worsen existing psychological distress. In addition, both physical and psychological impairments can lead to significant social problems. A number of interventions have been identified and used, some of them drawn from theoretical or conceptual frameworks and others based on research and/ or empirical testing. The most effective delivery of psychosocial health services should include the following components: identifying the population affected by psychosocial health issues who are most likely to be adversely affected by them; developing an appropriate plan for them; connecting and referring patients to appropriate psychosocial health services; giving them adequate support in managing their disease; coordinating psychosocial with biomedical healthcare; and ensuring appropriate follow-up so that the adequacy of interventions can be determined and changed, if necessary. ${ }^{13}$

Psychosocial interventions designed for this purpose should be divided into five categories: prevention, early detection, restoration, support and palliation. Firstly, preventive interventions are designed to avoid the development of predictable morbidity secondary to treatment and/ or disease. Secondly, early detection of patients' needs or problems refers to the assumption that early interventions' could have therapeutic results superior to those of delayed support, both for quality of life and survival. Thirdly, restorative interventions refer to actions used when a cure is likely, the aim being the control or elimination of residual cancer disability. Fourthly, supportive rehabilitation is planned to lessen disability related to chronic disease, characterized by cancer illness remission and progression. Lastly, palliation is required when curative treatments are likely to be ineffective and when maintaining or improving comfort becomes the main goal. These needs may be common to cancer patients in all cultures. Many patients, however, will not raise psychosocial concerns without prompting. Therefore, there is need to address an awareness of these concerns, willing to spend the time that is required for detection, evaluation and alleviation. The result is enhanced quality of life as the patient is studied in the domains of living that are important, extending across the continuum of care from diagnosis to palliative care. The important prevalence of psychosocial problems and psychiatric disturbances that have been reported in oncology, underlines the need for comprehensive psychosocial support for cancer patients and their families. Psychosocial support is designed to preserve, restore or enhance quality of life.

Psychological interventions are often multidisciplinary, with a variety of content. The type of psychological intervention ranges from information and education to more sophisticated support program including directive (behavioral or cognitive) therapies, or non-directive (dynamic or supportive) therapies. Social interventions usually include financial, household, equipment, and 
Psychosocial Care: Emerging Need for the Cancer Patients in Nepal

transport assistance depending on individual and family needs and resources. These interventions may be combined with the prescription of pharmacological (psychotropic, analgesic), physical, speech or occupational therapies, especially in rehabilitation program. Health care services devoted to delivery of these interventions are hospitals, hospices or homebased systems organized very differently depending on already available community resources and local practices. ${ }^{14}$ The literatures support the idea of a positive affect of social support and psychosocial wellbeing. ${ }^{15,16}$

Cancer affects everyone-the young and old, the rich and poor, men, women and children and represents a tremendous burden on patients, families and societies. Cancer is one of the leading causes of death in the world, particularly in developing countries due to expensive treatment, no health insurance system and lack of awareness, etc.

Developing countries like Nepal, where social exclusion is often a product of historical developments, including colonialism, involves the lack of resources, rights, goods and services, and the inability to participate in the normal relationships and activities, available to the majority of people in a society, whether in economic, social, cultural or political arenas. Nepal is a male dominated country, caste/ ethnic system still exists, the poor are becoming poorer and the rich becoming richer due to social and/or political discrimination. The effect of psychosocial intervention on the length of survival of cancer patients has been assessed in different studies and interventions and was found positively correlated with cancer survival. Quality of life refers not only to psychosocial distress and adjustment-related problems but also to the management of cancer symptoms and treatment of side-effects.

Psychotherapy may be the most critical intervention for the people whose psychological blocks may have contributed to or resulted due to their disease. If a belief that the person is unworthy of the positive things that life has to offer is embedded in his/her subconscious, then the person may not feel worthy of healing.

An extensive review of literatures revealed that a diagnosis of cancer can lead to feelings of anxiety, lack of control and presumptions of uncertain and insecure future. A strong social support network can buffer some of these feelings and successfully help the patient cope and progress through treatment and illness. Social support has been shown to exert a positive and an important influence on the patients adapting to the changes in their life that accompany a diagnosis of cancer. There is a strong need to implement psychosocial care concepts, besides the health facilities where diagnosis and treatment of cancer is being done. It is better to have counseling services and anonymous support groups to share their experiences with each other in specialized cancer hospitals as they required long term treatment and life long follow-up.

Cancer is a multi-factorial disease. For this reason, its treatment should take into consideration all factors, and not just the physical one. Research proves that a more holistic approach to cancer is more effective than the medical approach alone.

\section{REFERENCE}

1. World Health Organization. National cancer control programme: Policies and managerial guidelines. Geneva:WHO; 2002.

2. Parkin DM, Pisani P, Lopez AD, Masuyer E. At least one in seven cases of cancer is caused by smoking. Global estimates for 1985 . Int J Cancer 1994;59:494-504.

3. Pandey A. Cancer registry. Telecom conference. Kantipur news paper daily 2004 Mar 3.

4. Kleinman A. Suffering, Healing \& the Human Condition. New York, USA: Basic book publication; 1988. p. 18-30.

5. White CA. Psychosocial oncology. Psychiatry 2006;5(3):96-8.

6. Turner J, Kelly B, Swanson C, Allison R, Wetzig N. Psychosocial impact of newly diagnosed advanced breast cancer. Psychooncology. 2005 May;14(5):396-407.

7. Watson M, James-Roberts SI and Ashley S. Factors associated with emotional and behavioral problems among school age children of breast cancer patients. Br J Cancer 2006;94(1):43-50.

8. Grov EK, Dahl AA, \& Moum T. Anxiety, depression, and quality of life in caregivers of patients with cancer in late palliative phase. Ann Oncol 2005;16(7):1185-91.

9. Tatsuo A, Tomohito N, Nobuya A, Tatsuro N, EishoY, Hitoshi O, et al. Clinical Factors Associated with Suicidality in Cancer Patients. Japanese Journal of Clinical Oncology 2002;32:506-11.

10. Kantipur. Editorial. Kathmandu: Kantipur daily news paper; 2001 Jul 4.

11. DeVries AC, Glasper ER, and Detillion CE. Social modulation of stress response. Psychology and Behavior 2003;79:399-407.

12. Baum A and Posluszny D. Health psychology: Mapping biobehavioral contributors to health and illness. Annual Review of Psychology 1999;50:137-63.

13. House JS, Landis KR, and Umberson D. Social relationships and health. Science 1988;241:540-51.

14. Hurdle DE. Social support: A critical factor in women's health and health promotion. Health \& Social Work 2001;26:72-9.

15. WHO. Cancer [Online]. 2008 [Cited 2008 Feb 25]; Available from: URL: http://www.who.int/cancer/en/

16. Razavi D and Delvaux N. The psychiatrist's perspective on quality of life and quality of care in oncology: Concepts, symptom management, communication issues. European Journal of Cancer 1995;31(S5):25-9. 